\title{
Fuzzy Sub-Bi HX Group and its Bi Level Sub-Bi HX Groups
}

\author{
R.Muthuraj \\ Department of Mathematics \\ PSNA CET, Dindigul. - 624622
}

\author{
P.M.Sitharselvam \\ Department of Mathematics \\ PSNA CET, Dindigul. - 624622
}

\author{
K.H.Manikandan \\ Department of Mathematics \\ PSNA CET, Dindigul. - 624622
}

\begin{abstract}
The definition of a fuzzy HX group and define a new bialgebraic structure of fuzzy sub-bi HX group of a bi-hx group and some related properties are investigated and redefined in this paper.
\end{abstract}

\section{Keywords}

Bi HXgroup, Fuzzy set , fuzzy subgroup , fuzzy HX group, fuzzy sub-bi HX group of a bi HX group,

AMS Subject Classification (2000): 20N25, 03E72, 03F055, 06F35, 03G25.

\section{INTRODUCTION}

The concept of fuzzy sets was initiated by Zadeh. Then it has become a vigorous area of research in engineering, medical science, social science, graph theory etc. Rosenfeld gave the idea of fuzzy subgroups. Li Hongxing introduce the concept of HX group and the authors Luo Chengzhong, Mi Honghai , Li Hongxing introduce the concept of fuzzy HX group. The notion of bigroup was first introduced by P.L.Maggu in 1994. W.B. Vasantha Kandasamy and D.Meiyappan introduced concept of fuzzy sub-bigroup of a bigroup and fuzzy subbigroup of a group. In this paper we define a new bialgebraic structure of fuzzy sub-bi HX group of a bi HX group and study some of their related properties. we also discuss the image and pre image of a fuzzy sub-bi HX group of a bi HX group under homomorphism and anti-homomorphism.

\section{PRELIMINARIES}

In this section we site the fundamental definitions that will be used in the sequel. Through out this paper, $\quad \mathrm{G}=$ ( $\left.\mathrm{G},{ }^{*}\right)$ is a group, e is the identity element of $\mathrm{G}$, and $\mathrm{xy}$, we mean $\mathrm{x} * \mathrm{y}$.

\subsection{Definition}

In $2^{\mathrm{G}}-\{\phi\}$, a nonempty set $\vartheta \subset 2^{\mathrm{G}}-\{\phi\}$ is called a HX group on $\mathrm{G}$, if $\vartheta$ is a group with respect to the algebraic operation defined by $\mathrm{AB}=\{\mathrm{ab} / \mathrm{a} \in \mathrm{A}$ and $\mathrm{b} \in \mathrm{B}\}$, which its unit element is denoted by $\mathrm{E}$.

\subsection{Definition}

A set $(\vartheta,+, \bullet)$ with two binary operation + and $\bullet$ is called a bi HX group if there exist two proper subsets $\vartheta_{1}$ and $\vartheta_{2}$ of $\vartheta$ such that
A non-empty subset $\mathrm{H}$ of a bi $\mathrm{HX}$ group $(\vartheta,+, \bullet)$ is called a sub-bi HX group, if $\mathrm{H}$ itself is a bi HX group under ' + ' and ‘' operations defined on $\vartheta$.

\subsection{Definition}

Let $\mathrm{X}$ be any non empty set. A fuzzy subset $\lambda$ of $\mathrm{X}$ is a function $\lambda: X \rightarrow[0,1]$.

\subsection{Definition}

A fuzzy set $\lambda$ is called fuzzy HX subgroup of a HX group $\vartheta$ if for $A, B \in \vartheta$

\subsection{Definition}

$$
\begin{aligned}
& \text { (i) } \lambda(\mathrm{AB}) \geq \min \{\lambda(\mathrm{A}), \lambda(\mathrm{B})\} \\
& \text { (ii) } \lambda\left(\mathrm{A}^{-1}\right)=\lambda(\mathrm{A}) .
\end{aligned}
$$

Let $\lambda$ be a fuzzy HX subgroup of a HX group $\vartheta$. For any $\mathrm{t} \in[0,1]$, we define the set $\lambda_{\alpha}=\{\mathrm{A} \in \vartheta / \lambda(\mathrm{A}) \geq \alpha\}$ is called the level subset of $\lambda$.

\subsection{Definition}

Let $\vartheta=(\vartheta,+, \bullet)$ be a bi HX group. Then $\lambda: \vartheta \rightarrow$ $[0,1]$ is said to be fuzzy sub-bi HX group of the bi HX group $\vartheta$ if there exist two fuzzy subsets $\lambda_{1}$ of $\vartheta_{1}$ and $\lambda_{2}$ of $\vartheta_{2}$ such that

\subsection{Example}

$$
\begin{aligned}
& \text { i. } \lambda=\lambda_{1} \cup \lambda_{2} \\
& \text { ii. }\left(\lambda_{1},+\right) \text { is a fuzzy HX group of }\left(\vartheta_{1},+\right) \text {. } \\
& \text { iii. }\left(\lambda_{2}, \bullet\right) \text { is a fuzzy HX group of }\left(\vartheta_{2}, \bullet\right) \text {. }
\end{aligned}
$$

$$
\text { Let } G_{1}=\{0\} \text { and } G_{2}=\{1,-1\} \text {. }
$$

Then $\left(\mathrm{G}_{1},+\right)$ and $\left(\mathrm{G}_{2}, \bullet\right)$ are groups.

Let $\vartheta_{1}=\{\{0\}\}$ and $\vartheta_{2}=\{\{1\},\{-1\}\}$, then $\left(\vartheta_{1},+\right)$ and $\left(\vartheta_{2}, \bullet\right)$ are HX groups and hence $\vartheta=\left(\vartheta_{1} \cup \vartheta_{2},+, \bullet\right)$ is a bi HX group.

$$
\begin{array}{r}
\text { Define } \lambda_{1}: \vartheta_{1} \rightarrow[0,1] \text { by } \lambda_{1}(\{0\})=0.7 \text { and } \\
\lambda_{2}: \vartheta_{2} \rightarrow[0,1] \text { by } \lambda_{2}(\{1\})=0.8 \text { and } \\
\lambda_{2}(\{-1\})=0.5 .
\end{array}
$$

$$
\begin{aligned}
& \text { i. } \vartheta=\vartheta_{1} \cup \vartheta_{2} \\
& \text { ii. }\left(\vartheta_{1},+\right) \text { is a HX group. } \\
& \text { iii. }\left(\vartheta_{2}, \bullet\right) \text { is a HX group. }
\end{aligned}
$$




\subsection{Definition}

Let $\vartheta=\left(\vartheta_{1} \cup \vartheta_{2},+, \bullet\right)$ and $\vartheta^{\prime}=\left(\vartheta^{\prime}{ }_{1} \cup \vartheta^{\prime}{ }_{2}, \oplus, \mathrm{o}\right)$ be any two bi HX groups. We say the map $f: \vartheta \rightarrow \vartheta^{\prime}$ is said to be a bi HX group homomorphism if $\mathrm{f}$ restricted to $\vartheta_{1}$ (that is , $\mathrm{f} / \vartheta_{1}$ ) is a $\mathrm{HX}$ group homomorphism from $\vartheta_{1}$ to $\vartheta^{\prime}{ }_{1}$ and $\mathrm{f}$ restricted to $\vartheta_{2}$ (i.e. f $/ \vartheta_{2}$ ) is a HX group homomorphism from $\vartheta_{2}$ to $\vartheta_{2}^{\prime}$

\section{BI-LEVEL SUBSETS OF A FUZZY SUB-BI HX GROUP OF A BI HX GROUP 3.1 Definition}

Let $\vartheta=\left(\vartheta_{1} \cup \vartheta_{2},+, \bullet\right)$ be a bi HX group and $\lambda=\left(\lambda_{1} \cup \lambda_{2},+, \bullet\right)$ be a fuzzy sub-bi HX group of the bi HX group $\vartheta$. The bi-level subset of the fuzzy sub-bi HX group $\lambda$ of the bi HX group $\vartheta$ is defined as $\lambda_{\alpha}=\lambda_{1 \alpha} \cup \lambda_{2 \alpha}$ for every $\alpha \in\left[0, \min \left\{\lambda_{1}\left(\mathrm{E}_{1}\right), \lambda_{2}\left(\mathrm{E}_{2}\right)\right\}\right]$ where $\mathrm{E}_{1}$ denotes the identity element of the HX group $\left(\vartheta_{1},+\right)$ and $E_{2}$ denotes the identity element of the HX group $\left(\vartheta_{2}, \bullet\right)$.

\section{Remark:}

The condition $\alpha \in\left[0, \min \left\{\lambda_{1}\left(E_{1}\right), \lambda_{2}\left(E_{2}\right)\right\}\right]$ is essential for the bi-level subset to be a sub-bi $\mathrm{HX}$ group, for if $\alpha \notin\left[0, \min \left\{\lambda_{1}\left(E_{1}\right), \lambda_{2}\left(E_{2}\right)\right\}\right]$, the bi- level subset need not in general be a sub-bi HX group of the bi HX group $\vartheta$.

\subsection{Example}

Consider Example 2.1, the bi-level subset $\lambda_{\alpha}$ for $\alpha=0.8$ of the fuzzy sub-bi HX group $\lambda$ is given by $\lambda_{\alpha}=\{1\}$ which is not a sub-bi HX group of the bi HX group $\vartheta$. Therefore the bi-level subset $\lambda_{\alpha}$, for $\alpha=0.8$ is not a sub-bi HX group of the bi HX group $\vartheta$.

\subsection{Theorem}

Every bi-level subset of a fuzzy sub-bi HX group $\lambda$ of a bi HX group $\vartheta$ is a sub-bi HX group of the bi HX group $\vartheta$.

\section{Proof}

Let $\lambda=\left(\lambda_{1} \cup \lambda_{2},+, \bullet\right)$ be a fuzzy sub-bi HX group of a bi HX group $\vartheta=\left(\vartheta_{1} \cup \vartheta_{2},+, \bullet\right)$. Consider the bilevel subset $\lambda_{\alpha}$ of a fuzzy sub-bi HX group $\lambda$ for every $\alpha \in\left[0, \min \left\{\lambda_{1}\left(E_{1}\right), \lambda_{2}\left(E_{2}\right)\right\}\right]$ where $E_{1}$ denotes the identity element of the HX group $\left(\vartheta_{1},+\right)$ and $E_{2}$ denotes the identity element of the HX group $\left(\vartheta_{2}, \bullet\right)$.

Then $\lambda_{\alpha}=\lambda_{1 \alpha} \cup \lambda_{2 \alpha}$ where $\lambda_{1 \alpha}$ and $\lambda_{2 \alpha}$ are sub HX groups of $\vartheta_{1}$ and $\vartheta_{2}$ respectively. Hence by the definition of sub-bi HX group $\lambda_{\alpha}$ is a sub-bi HX group of the bi HX $\operatorname{group}(\vartheta,+, \bullet)$.

\subsection{Theorem}

Let $\vartheta$ be a bi HX group and $\lambda$ be a fuzzy sub-bi HX group of $\vartheta$. Two bi-level subgroups $\lambda_{\alpha}, \lambda_{\beta}$ with $\alpha<\beta$ of $\lambda$ are equal if and only if there is no $A \in \vartheta$ such that $\alpha \leq \lambda(\mathrm{A})<\beta$.
Proof

Let $\lambda_{\alpha}=\lambda_{\beta}$.

Suppose that there exists $\mathrm{A} \in \vartheta$ such that $\alpha<\lambda(\mathrm{A})<\beta$ then, $\quad \lambda_{\beta} \subset \lambda_{\alpha}$. Since $A \in \lambda_{\alpha}$ but not in $\lambda_{\beta}$ which contradicts the hypothesis. Hence there exists no $A \in \vartheta$ such that $\alpha \leq \lambda(\mathrm{A})<\beta$.

Conversely, let there be no $\mathrm{A} \in \vartheta$ such that $\alpha \leq \lambda(\mathrm{A})<\beta$.

Since $\alpha<\beta$, we have, $\lambda_{\beta} \subseteq \lambda_{\alpha}$.

Let $\mathrm{A} \in \lambda_{\alpha}$, then $\lambda(\mathrm{A}) \geq \alpha$.

Since there exists no $A \in \vartheta$ such that $\alpha \leq \lambda(A)<\beta$, we have $\lambda(\mathrm{A}) \geq \beta \quad$ which implies $\quad \lambda_{\alpha} \subseteq \lambda_{\beta}$.

Hence $\lambda_{\alpha}=\lambda_{\beta}$.

\subsection{Theorem}

Let $\vartheta$ be a bi HX group. Let $\lambda$ be a fuzzy subset of $\vartheta$ such that the bi-level subset $\lambda_{\alpha}=\lambda_{1 \alpha} \cup \lambda_{2 \alpha}$ is a sub-bi HX group of the bi HX group $\vartheta$ where $\alpha \in\left[0, \min \left\{\mu_{\mathrm{A} 1}\left(\mathrm{E}_{1}\right), \mu_{\mathrm{A} 2}\left(\mathrm{E}_{2}\right)\right\}\right]$, then $\lambda$ is a fuzzy sub-bi HX group of $\vartheta$.

Proof

$$
\text { Let } \vartheta=\left(\vartheta_{1} \cup \vartheta_{2},+, \bullet\right) \text { be a bi HX group. }
$$

Let the bi-level subset $\lambda_{\alpha}$ is a sub-bi HX group of the bi HX group $\vartheta$. Then $\lambda_{\alpha}=\lambda_{1 \alpha} \cup \lambda_{2 \alpha}$ and $\left(\lambda_{1 \alpha},+\right)$ is a sub HX group of $\left(\vartheta_{1},+\right)$ and $\left(\lambda_{2 \alpha}, \bullet\right)$ is a sub HX group of $\left(\vartheta_{2}, \bullet\right)$. We have to prove that $\lambda$ is a fuzzy sub-bi HX group of the bi HX group $\vartheta$.

$\left(\lambda_{1 \alpha},+\right)$ is a sub HX group of $\left(\vartheta_{1},+\right)$, then $\left(\lambda_{1},+\right)$ is a fuzzy sub HX group of $\left(\vartheta_{1},+\right)$.

$\left(\lambda_{2 \alpha}, \bullet\right)$ is a sub HX group of $\left(\vartheta_{2}, \bullet\right)$, then $\left(\lambda_{2}, \bullet\right)$ is a fuzzy sub HX group of $\left(\vartheta_{2}, \bullet\right)$.Clearly $\lambda=\lambda_{1} \cup \lambda_{2}$.

This implies that $\lambda$ is a fuzzy sub-bi HX group of the bi HX group $\vartheta$.

\subsection{Theorem}

A fuzzy subset $\lambda$ of $\vartheta$ is a fuzzy sub-bi HX group of a bi HX group $\vartheta$ if and only if the bi-level subsets $\lambda_{\alpha}$, $\alpha \in\left[0, \min \left\{\lambda\left(\mathrm{E}_{1}\right), \lambda\left(\mathrm{E}_{2}\right)\right\}\right]$, are sub-bi HX groups of $\vartheta$.

\section{Proof}

$$
\text { It is clear. }
$$

\section{Remark}

As a consequence of the Theorem 3.2, the bi-level subbi HX groups of a fuzzy sub-bi HX group $\lambda$ of a bi HX group $\vartheta$ form a chain. Since $\lambda(A) \leq \lambda\left(E_{1}\right)$ or $\lambda(A) \leq \lambda\left(E_{2}\right)$ for all $\lambda$ in $\vartheta$. Therefore, $\lambda_{\alpha 0}, \alpha_{0} \in\left[0, \min \left\{\lambda\left(E_{1}\right), \lambda\left(E_{2}\right)\right\}\right]$, where $\alpha_{0}=\min \left\{\lambda\left(E_{1}\right), \lambda\left(E_{2}\right)\right\}$ is the smallest sub-bi HX group and we have the chain :

$\left\{\mathrm{E}_{1}, \mathrm{E}_{2}\right\} \subseteq \lambda_{\alpha 0} \subset \lambda_{\alpha 1} \subset \lambda_{\alpha 2} \subset \lambda_{\alpha 3} \subset \ldots \ldots \subset \lambda_{\alpha \mathrm{n}}$, where $\alpha_{0}>\alpha_{1}>\alpha_{2}>\ldots .>\alpha_{n}$. 


\section{FUZZY SUB-BI HX GROUP OF A BI HX GROUP UNDER HOMOMORPHISM AND ANTI HOMOMORPHISM}

We now discuss the properties of a fuzzy sub-bi HX group of a bi HX group under homomorphism and anti homomorphism.

\subsection{Theorem}

Let $\mathrm{f}$ be a homomorphism from a bi HX group $\vartheta$ into a bi HX group $\vartheta^{\prime}$. If $\lambda$ is a fuzzy sub-bi HX group of $\vartheta$ and $\lambda$ is $f$-invariant, then $f(\lambda)$, the image of $\lambda$ under $f$, is a fuzzy sub-bi HX group of $\vartheta^{\prime}$

\section{Proof}

Let $\vartheta=\left(\vartheta_{1} \cup \vartheta_{2},+, \bullet\right)$ and $\vartheta^{\prime}=\left(\vartheta^{\prime}{ }_{1} \cup \vartheta^{\prime}{ }_{2}, \oplus, o\right)$ be any two bi HX groups. Let $\lambda=\left(\lambda_{1} \cup \lambda_{2},+, \bullet\right)$ be a fuzzy sub-bi HX group of a bi HX group $\vartheta$. Then $\left(\lambda_{1},+\right)$ is a fuzzy sub HX group of $\left(\vartheta_{1},+\right)$ and $\left(\lambda_{2}, \bullet\right)$ is a fuzzy sub HX group of $\left(\vartheta_{2}, \bullet\right)$.

Let $f: \vartheta \rightarrow \vartheta^{\prime}$ be a homomorphism.

That is,$f(A B)=f(A) f(B)$ for all $A, B$ in $\vartheta$.

We have to prove that $f(\lambda)=\eta$ is a fuzzy sub-bi HX group of $\vartheta^{\prime}$.

$\eta=\mathrm{f}(\lambda)=\mathrm{f}\left(\lambda_{1} \cup \lambda_{2}\right)=\mathrm{f}\left(\lambda_{1}\right) \cup \mathrm{f}\left(\lambda_{2}\right)$.

Since $\mathrm{f}$ is a homomorphism, $\left(\mathrm{f}\left(\lambda_{1}\right), \oplus\right)$ is a fuzzy sub HX group of $\left(\vartheta^{\prime}{ }_{1}, \oplus\right)$ and $\left(f\left(\lambda_{2}\right), o\right)$ is a fuzzy sub HX group of $\left(\vartheta_{2}^{\prime}, 0\right)$.

Hence $\eta=f\left(\lambda_{1}\right) \cup f\left(\lambda_{2}\right)$ is a fuzzy sub-bi HX group of $\vartheta^{\prime}$.

\subsection{Theorem}

The homomorphic pre-image of a fuzzy sub-bi HX group $\eta$ of a bi HX group $\vartheta^{\prime}$ is a fuzzy sub-bi HX group of a bi HX group $\vartheta$.

Proof

Let $\vartheta=\left(\vartheta_{1} \cup \vartheta_{2},+, \bullet\right)$ and $\vartheta^{\prime}=\left(\vartheta^{\prime}{ }_{1} \cup \vartheta^{\prime}{ }_{2}, \oplus, o\right)$ be any two bi HX groups.

Let $\eta=\left(\eta_{1} \cup \eta_{2}, \oplus\right.$, o $)$ be a fuzzy sub-bi HX group of a bi HX group $\vartheta^{\prime}$. Then $\left(\eta_{1}, \oplus\right)$ is a fuzzy sub HX group of $\left(\vartheta^{\prime}{ }_{1}, \oplus\right)$ and $\left(\eta_{2}, \mathrm{O}\right)$ is a fuzzy sub HX group of $\left(\vartheta^{\prime}{ }_{2}, \mathrm{O}\right)$.

Let $\mathrm{f}: \vartheta \rightarrow \vartheta^{\prime}$ be a homomorphism.

That is , $f(A B)=f(A) f(B)$ for all $A, B$ in $\vartheta$.

We have to prove that $f^{-1}(\eta)=\lambda$ is a fuzzy sub-bi HX group of $\vartheta$.

$\lambda=\mathrm{f}^{-1}(\eta)=\mathrm{f}^{-1}\left(\eta_{1} \cup \eta_{2}\right)=\mathrm{f}^{-1}\left(\eta_{1}\right) \cup \mathrm{f}^{-1}\left(\eta_{2}\right)$.

Since $\mathrm{f}$ is a homomorphism, $\left(\mathrm{f}^{-1}\left(\eta_{1}\right),+\right)$ is a fuzzy sub HX group of $\left(\vartheta_{1},+\right)$ and $\left(f^{-1}\left(\eta_{2}\right), \bullet\right)$ is a fuzzy sub HX group of $\left(\vartheta_{2}, \bullet\right)$.

Hence $\lambda=\mathrm{f}^{-1}\left(\eta_{1}\right) \cup \mathrm{f}^{-1}\left(\eta_{2}\right)$ is a fuzzy sub-bi HX group of $\vartheta$

\subsection{Theorem}

Let $\mathrm{f}$ be an anti homomorphism from a bi HX group $\vartheta$ into a bi HX group $\vartheta^{\prime}$. If $\lambda$ is a fuzzy sub-bi HX group of $\vartheta$ and $\lambda$ is f-invariant, then $f(\lambda)$, the image of $\lambda$ under $f$, is a fuzzy sub-bi HX group of $\vartheta^{\prime}$
Proof

Let $\vartheta=\left(\vartheta_{1} \cup \vartheta_{2},+, \bullet\right)$ and $\vartheta^{\prime}=\left(\vartheta^{\prime}{ }_{1} \cup \vartheta^{\prime}{ }_{2}, \oplus, o\right)$ be any two bi HX groups. Let $\lambda=\left(\lambda_{1} \cup \lambda_{2},+, \bullet\right)$ be a fuzzy sub-bi HX group of a bi HX group $\vartheta$. Then $\left(\lambda_{1},+\right)$ is a fuzzy sub HX group of $\left(\vartheta_{1},+\right)$ and $\left(\lambda_{2}, \bullet\right)$ is a fuzzy sub HX group of $\left(\vartheta_{2}, \bullet\right)$.

Let $f: \vartheta \rightarrow \vartheta^{\prime}$ be an anti homomorphism.

That is,$f(A B)=f(B) f(A)$ for all $A, B$ in $\vartheta$.

We have to prove that $f(\lambda)=\eta$ is a fuzzy sub-bi HX group of $\vartheta^{\prime}$.

$\eta=\mathrm{f}(\lambda)=\mathrm{f}\left(\lambda_{1} \cup \lambda_{2}\right)=\mathrm{f}\left(\lambda_{1}\right) \cup \mathrm{f}\left(\lambda_{2}\right)$.

Since $f$ is an anti homomorphism, $\left(\mathrm{f}\left(\lambda_{1}\right), \oplus\right)$ is a fuzzy sub HX group of $\left(\vartheta^{\prime}{ }_{1}, \oplus\right)$ and $\left(\mathrm{f}\left(\lambda_{2}\right), \mathrm{O}\right)$ is a fuzzy sub HX group of $\left(\vartheta^{\prime}{ }_{2}, \mathrm{o}\right)$.

Hence $\eta=f\left(\lambda_{1}\right) \cup f\left(\lambda_{2}\right)$ is a fuzzy sub-bi HX group of $\vartheta^{\prime}$.

\subsection{Theorem}

The anti homomorphic pre-image of a fuzzy sub-bi HX group $\eta$ of a bi HX group $\vartheta^{\prime}$ is a fuzzy sub-bi HX group of a bi HX group $\vartheta$.

\section{Proof}

Let $\vartheta=\left(\vartheta_{1} \cup \vartheta_{2},+, \bullet\right)$ and $\vartheta^{\prime}=\left(\vartheta^{\prime}{ }_{1} \cup \vartheta^{\prime}{ }_{2}, \oplus, o\right)$ be any two bi HX groups.

Let $\eta=\left(\eta_{1} \cup \eta_{2}, \oplus, o\right)$ be a fuzzy sub-bi HX group of a bi HX group $\vartheta^{\prime}$.Then $\left(\eta_{1}, \oplus\right)$ is a fuzzy sub HX group of $\left(\vartheta^{\prime}{ }_{1}, \oplus\right)$ and $\left(\eta_{2}, \mathrm{o}\right)$ is a fuzzy sub HX group of $\left(\vartheta^{\prime}{ }_{2}, \mathrm{o}\right)$.

Let $\mathrm{f}: \vartheta \rightarrow \vartheta^{\prime}$ be an anti homomorphism.

That is, $\mathrm{f}(\mathrm{AB})=\mathrm{f}(\mathrm{B}) \mathrm{f}(\mathrm{A})$ for all $\mathrm{A}, \mathrm{B}$ in $\vartheta$.

We have to prove that $f^{-1}(\eta)=\lambda$ is a fuzzy sub-bi HX group of $\vartheta$.

$\lambda=\mathrm{f}^{-1}(\eta)=\mathrm{f}^{-1}\left(\eta_{1} \cup \eta_{2}\right)=\mathrm{f}^{-1}\left(\eta_{1}\right) \cup \mathrm{f}^{-1}\left(\eta_{2}\right)$.

Since $\mathrm{f}$ is an anti homomorphism, $\left(\mathrm{f}^{-1}\left(\eta_{1}\right),+\right)$ is a fuzzy sub HX group of $\left(\vartheta_{1},+\right)$ and $\left(f^{-1}\left(\eta_{2}\right), \bullet\right)$ is a fuzzy sub HX group of $\left(\vartheta_{2}, \bullet\right)$.

Hence $\lambda=\mathrm{f}^{-1}\left(\eta_{1}\right) \cup \mathrm{f}^{-1}\left(\eta_{2}\right)$ is a fuzzy sub-bi HX group of 9

\section{CONCLUSION}

In this paper, we define a new bialgebraic structure of fuzzy sub-bi Hx group and studied some of its properties.Futher, we wish to define the relation between fuzzy (normal) HX group and fuzzy (normal) sub bi HX group in HX group also the same in Intuitionistic fuzzy and other some groups are in progress.

\section{REFERENCES}

[1] Choudhury.F.P. and Chakraborty.A.B. and Khare.S.S., A note on fuzzy subgroups and fuzzy homomorphism, Journal of mathematical analysis and applications 131, $537-553(1988)$.

[2] Das. P.S, Fuzzy groups and level subgroups, J.Math.Anal. Appl, 84 (1981) 264-269.

[3] Dixit.V.N., Rajesh Kumar, Naseem Ajmal., Level subgroups and union of fuzzy subgroups, Fuzzy Sets and Systems, 37, 359-371 (1990). 
[4] Li Hongxing, HX group, BESEFAL,33(1987), pp(31-37).

[5] Luo Chengzhong, Mi Honghai, Li Hongxing , Fuzzy HX group , BUSEFAL.

[6] Mehmet sait EROGLU, The homomorphic image of a fuzzy subgroup is always a Fuzzy subgroup, Fuzzy sets and Systems, 33 (1989) 255 - 256.

[7] Mohamed Asaad, Groups and Fuzzy subgroups, Fuzzy sets and systems 39(1991) 323-328.

[8] Mustafa Akgul, Some properties of fuzzy groups, Journal of mathematical analysis and applications 133, 93-100 (1988)
[9] Muthuraj.R., Sithar Selvam.P.M., Muthuraman.M.S., Anti Q-fuzzy group and its lower Level subgroups, International journal of Computer Applications (09758887), Volume 3- no.3, June 2010, 16-20.

[10] Palaniappan.N., Muthuraj.R., , Anti fuzzy group and Lower level subgroups, Antartica J.Math., 1 (1) (2004), 71-76.

[11] Prabir Bhattacharya, Fuzzy Subgroups: Some Characterizations,J.Math. Anal. Appl.128 (1987) 241 252.

[12] Rajesh kumar, Homomorphism and fuzzy (fuzzy normal) subgroups, Fuzzy sets and Systems, 44 (1991) $165-168$.

[13] Rosenfeld.A., fuzzy groups, J. math. Anal.Appl. 35 (1971), 512-517. 